\title{
Frontal cortex-like functions of the subthalamic nucleus
}

\author{
Christelle Baunez ${ }^{1,2}$ * and Sylvie Lardeux ${ }^{1,2+}$ \\ 1 Laboratoire de Neurobiologie de la Cognition, UMR6155 CNRS, Université de Provence Marseille, Marseille, Frances \\ 2 Pôle3C, UMR6155, Université de Provence, Marseille, France
}

\author{
Edited by: \\ Elizabeth Abercrombie, \\ Rutgers-Newark: The State University \\ of New Jersey, USA \\ Reviewed by: \\ Andrew M. Farrar, University at \\ Buffalo, The State University of New \\ York, USA \\ Verity Brown, University of St. \\ Andrews, UK \\ *Correspondence: \\ Christelle Baunez, Laboratoire de \\ Neurobiologie de la Cognition, \\ UMR6155 CNRS, Aix-Marseille \\ Université, Marseille, France. \\ e-mail: christelle. \\ baunez@univ-provence.fr \\ ${ }^{t}$ Present address: \\ Sylvie Lardeux, Department of \\ Psychiatry, Albert Einstein College of \\ Medicine, Bronx, NY, USA.
}

The subthalamic nucleus (STN) has been considered a motor structure for a long time. Over the last 20 years, anatomical and behavioral data have highlighted the position of the STN within a prefrontal-associative and a limbic loops, suggesting that the STN should play a critical role in frontal functions such as attention, inhibitory control (including impulsive action, compulsivity, impulsive choice), and motivation. Here we will review the work highlighting these functions of the STN.

Keywords: basal ganglia, subthalamic nucleus, deep brain stimulation, attention, motivation, impulsivity, compulsion, behavioral inhibition

\section{INTRODUCTION}

When studying the basal ganglia, many researchers focus their experiments on the so-called motor loop, one of the five loops described by Alexander et al. (1986). In this motor loop, the subthalamic nucleus (STN) belongs to the indirect pathway, positioned as a relay between the external and the internal globus pallidus (GPe and GPi respectively). Its dysregulation in the case of parkinsonism lead to the suggestion of STN inactivation as an alternative strategy to dopaminergic treatments. STN high frequency stimulation (HFS) was therefore introduced (Limousin et al., 1995) and is currently very successful in treating the motor symptoms of Parkinson's disease (PD). More recently, the STN has also been described as a possible input structure to the basal ganglia since it receives a direct projection from the cortex, a pathway now called the "hyperdirect pathway" (Nambu et al., 2002). When looking at the classical diagrams of the five parallel loops, the STN is traditionally drawn only on the motor loop, but anatomical data indicate that it should not be omitted from the other loops, including the associative and limbic circuits. Indeed, the STN receives and sends projections from/to the ventral pallidum (VP) and also receives inputs from the prefrontal cortex (Groenewegen and Berendse, 1990; Groenewegen et al., 1993; Maurice et al., 1998a,b, 1999). Given these connections, it is expected that its

Abbreviations: 5-CSRTT, 5-choice serial reaction time task; CS, conditioned stimulus; DBS, deep brain stimulation; FR1, fixed ratio 1; GPe/i, external/internal segment of the globus pallidus; HFS, high frequency stimulation; OCD, obsessive compulsive disorder; OFC, orbito-frontal cortex; PD, Parkinson's disease; PET, positron emission tomography; RT, reaction time; STN, subthalamic nucleus; US, unconditioned stimulus; VP, ventral pallidum. functional involvement should not be limited to motor functions, but should also include "frontal functions," like attention, inhibition control, and motivation. Some aspects of them have been partially reviewed (Temel et al., 2005), but there are now more experimental and clinical sets of data supporting this involvement of STN in frontal-like functions, and especially on motivation.

\section{STN IN ATTENTION AND IMPULSIVITY STN IN ATTENTION}

The role of the STN in arousal, wakefulness, and alertness was first suggested in the late 1960s based on studies carried out in the cat (Naquet et al., 1966; Lindsley et al., 1970). This line of research was abandoned until the resurgence of interest in the STN as a therapeutic target for the treatment of PD in the 1990s (Bergman et al., 1990; Benazzouz et al., 1993).

In a study assessing the effects of STN lesions on the performance of a simple reaction time (RT) task, we have shown that STN lesions induce a dramatic increase in the number of premature responses (i.e., early withdrawal of the paw from a lever, before the onset of the visual trigger stimulus). This deficit was hypothesized to result from either an attentional deficit or disinhibition (Baunez et al., 1995).

In order to further assess attentional functions in rats, we have then used the "5-choice serial RT task" (5-CSRTT) in which the animals are trained to wait and detect a brief visual stimulus that is randomly presented in five possible different locations. The animals have to divide their attention between these five possible choices and then respond by making a nose-poke in the appropriate location in order to obtain a food reward. The 
measure of accuracy is considered as an index of attentional performance (Robbins, 2002). In this specific visual attentional task, we have shown that bilateral excitotoxic lesions of the STN seriously impaired accuracy, thus suggesting an attentional deficit (Baunez and Robbins, 1997). These results were the first to highlight the involvement of STN in cognitive functions. Decreased accuracy in the same task was also observed after pharmacological inactivation of the STN induced by stimulation of GABA receptors with muscimol (Baunez and Robbins, 1999). Interestingly enough, bilateral STN HFS did not reproduce the severe deficit on accuracy in the 5CSRTT, but only induced a transient deficit (Baunez et al., 2007). This difference between STN lesion and HFS suggests that HFS does not truly mimic an inactivation of the structure, as suggested by human imaging data (for example (Hershey et al., 2003).

We provided the first evidence of a functional role for the hyperdirect pathway in the attentional deficits observed in this behavioral task by disconnecting the medial prefrontal cortex and the STN, through lesioning the prefrontal cortex on one side and the STN on the contralateral side. Indeed, the disconnection induced similar deficits to those observed after bilateral STN lesions (Chudasama et al., 2003).

From our rat data, the STN therefore appears to be involved in attentional processes, possibly via the hyperdirect pathway. Clinical studies assessing the cognitive functions of PD patients who underwent STN deep brain stimulation (DBS) showed contrasting results. Some of those also report some attentional decline (SaintCyr et al., 2000; Mallet et al., 2007; York et al., 2008), while others report improvements (Fimm et al., 2009), or no further decline (Ardouin et al., 1999; Brusa et al., 2001). Unfortunately, these studies rarely used tasks specifically designed to assess attention. Furthermore, it is important to note that human data are mostly provided by clinical studies in PD patients, therefore depleted in DA. The effects of STN manipulation in a DA depleted brain may therefore not always match animal studies carried out in intact subjects. However, when STN HFS was applied in DA depleted rats performing the attentional task, there was no detrimental effect of STN HFS on the measure of visual attention (i.e., accuracy; Baunez et al., 2007).

\section{STN AND INHIBITION CONTROL}

The control of inhibition is commonly associated with impulsivity and compulsive behavior. Impulsivity itself can be dissociated into various aspects: impulsive action and impulsive choice (Eagle and Baunez, 2010). The effects of STN manipulations have been studied on these various forms by means of different behavioral procedures.

\section{Impulsive action}

As previously mentioned, we have observed increased premature responding after STN lesions in a simple RT task. This deficit may reflect a lack of inhibitory control (Baunez et al., 1995). This result has been replicated after a unilateral STN lesion (Phillips and Brown, 1999).

Premature responses can also be measured in the visual attentional task, the 5-CSRTT, when the animals are unable to wait for the brief visual stimulus, but respond in any hole before the onset of the light. In this task, we have shown that bilateral lesions of STN increase premature responses (Baunez and Robbins, 1997). Reversible inactivation of the STN by intra-STN muscimol injections induced the same effect (Baunez and Robbins, 1999). Surprisingly, here again, although HFS is supposed to mimic an inactivation of the stimulated target, bilateral STN HFS did not increase the number of premature responses (Baunez et al., 2007), a measure that has been shown to be amplitude and frequency dependent in rats performing a RT task (Desbonnet et al., 2004). This latter observation confirms that STN HFS may not inactivate the STN in the same manner as the lesion or muscimol injections do. However STN HFS applied in PD patients was shown to impair response inhibition in a go/no go task (Ballanger et al., 2009), in line with our lesion studies and in line with the computational model suggesting that the STN could play a critical role in "holding a response" (Frank et al., 2007). A more recent study confirmed this effect of STN HFS on go-no go performance but provided further details, showing that the limbic territory of STN (i.e., ventral) was more responsible for the control of inhibition than the motor territory (i.e., dorsal; Hershey et al., 2010). Although the functional territories are clearly identified and have been functionally dissociated in humans (Mallet et al., 2007), it is difficult to address this issue in the rodents and all the studies currently available in the rat do not allow discussion of the functions of the STN in terms of anatomical territories.

Many manipulations have been tested in the 5-CSRTT and reviewing these numerous studies (Eagle and Baunez, 2010) lead to the generation of a schematic diagram illustrated in Figure 1, in which each lesion that induces increased premature responding are highlighted. These structures include the infra-limbic cortex, orbito-frontal cortex (OFC), dorso-medial striatum, and the STN. We have also added the cingulate cortex, the nucleus accumbens (core and shell), as pharmacological treatment or manipulation of the task can also increase premature responding in animals with damage to these regions.

The premature responses measured in RT and 5-CSRT tasks, or incorrect responses in a go/no go task reflect the inability to inhibit the initiation of a response, including the selection of the action to perform and waiting for the trigger signal. In contrast, impulse control is often studied in human subjects using a stop task. However, it is becoming clear that the stop task, by measuring the speed to stop an ongoing action, involves different inhibitory processes which depend on different neural mechanisms (Rubia et al., 2001; Eagle et al., 2008a).

Using this task, it was indeed shown in human subjects that STN activation was correlated with faster stopping abilities (Aron and Poldrack, 2006). In line with this observation, using a stopsignal RT task in the rat, we have shown that STN lesions impair animals' ability to stop an action once it has been initiated (Eagle et al., 2008b). Again, we have synthesized the data obtained in the rat on the schematic diagram illustrated in Figure 1. This figure highlights again an interesting set of cerebral structures: the OFC, the dorso-medial striatum, as well as the STN.

\section{Perseverative behavior-compulsivity}

Another form of deficit in response inhibition relates to repetition of a response, even if it might be an inappropriate response. This is also called compulsive behavior and is often linked with 
addiction when the drug consumption switches from recreational use to compulsive use. When testing the effects of bilateral STN lesions in the 5-CSRTT, we have observed an increased level of perseverative responses toward the response locations and toward the magazine where the animals collect the food reward. These results suggest a deficit in response control and an increased level of motivation for the reward (discussed in the next paragraph; Baunez and Robbins, 1997). Interestingly, the perseverative responses on both response location and reward magazine were also observed after bilateral STN HFS (Baunez et al., 2007) and after pharmacological inactivation of the STN (Baunez and Robbins, 1999). Increased perseverative responses in the 5-CSRTT have been also observed after lesion of the pre-limbic cortex, the OFC, the dorso-medial striatum, and the nucleus accumbens core (after failed trials; Eagle and Baunez, 2010), as shown in Figure 2.

\section{Impulsive choice}

Impulsive choice is traditionally assessed using the delaydiscounting task. In this task, the animals are given the choice between a small but immediate reward and a large but delayed reward. When tested in this procedure, the STN-lesioned animals were surprisingly able to overcome their impulsivity and wait for a bigger reward (Winstanley et al., 2005), in line with the enhanced incentive motivation observed after STN lesions (Baunez et al., 2002). This result suggests that when impulsivity competes with motivation, motivation for a bigger reward wins in STN-lesioned

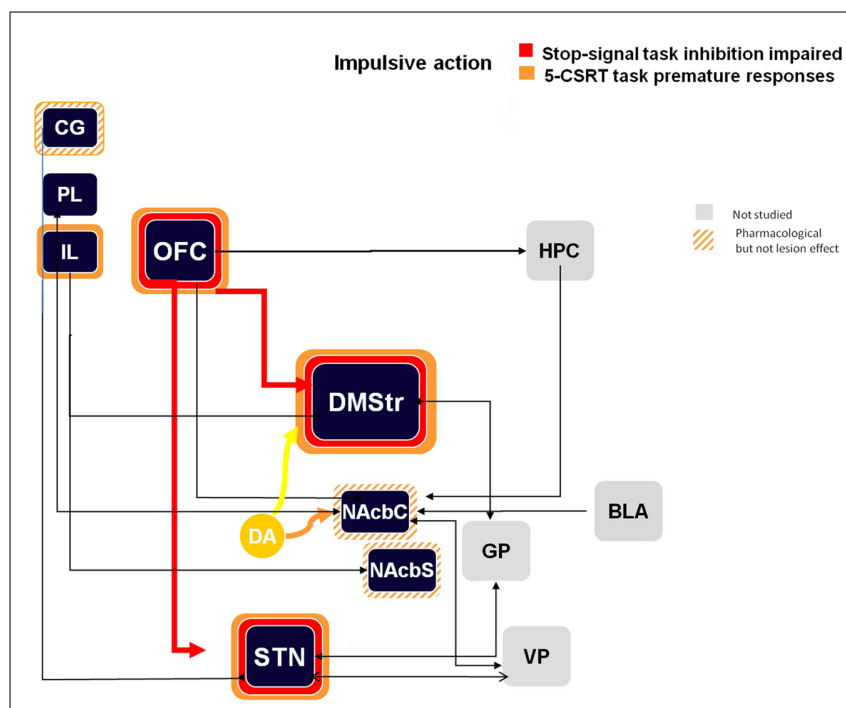

FIGURE 1 | Schematic representation of the impulsive action processes in the stop task and the 5-CSRTT (premature responses) from lesion and pharmacological manipulations. Colored bands surrounding each structure highlight the impulsive-like effect of the lesion of the given structure. Hatched-shaded bands indicate no effect of excitotoxic lesion, but an effect under pharmacological manipulation or other conditions). Gray structures indicate that no information is available. Arrows materialize anatomical connections between regions. CG, cingulate cortex; PL, pre-limbic cortex; IL, infra-limbic cortex; OFC, orbito-frontal cortex; HPC, hippocampus; DMStr, dorso-medial striatum; NAcbC, nucleus accumbens core; NAcbS, nucleus accumbens shell; GP, globus pallidus; BLA, basolateral amygdale; STN, subthalamic nucleus; VP, ventral pallidum. rats. This dissociation between impulsive action enhanced by STN lesions and decreased impulsive choice was confirmed by another study (Uslaner and Robinson, 2006). These results suggest a specific role of STN in the control of inhibition that can be under the influence of the motivation for the outcome (Eagle and Baunez, 2010).

Again, although there are discrepant results regarding the effects of OFC, Figure 3 illustrates commonalities of effects between the OFC, the dorso-medial striatum, and the STN.

\section{Conclusion}

Taken together these deficits reported on various forms of inhibitory control highlight a potential role for the STN in impulse control disorders. In line with this hypothesis, STN HFS has been shown to have beneficial effects in patients suffering from obsessive compulsive disorder (OCD; Mallet et al., 2008). The network involving the OFC, the dorso-medial striatum, and the STN highlighted in Figure 3 seems to be critical for certain forms of impulsivity/compulsivity. Interestingly, a recent study using PET imaging suggests that the therapeutic effect of STN HFS in OCD patients is related to a decrease in prefrontal cortex metabolism (Le Jeune et al., 2010).

\section{STN IN MOTIVATIONAL PROCESSES}

The first observation that the STN may be involved in motivational processes comes from behavioral studies of STN lesions in

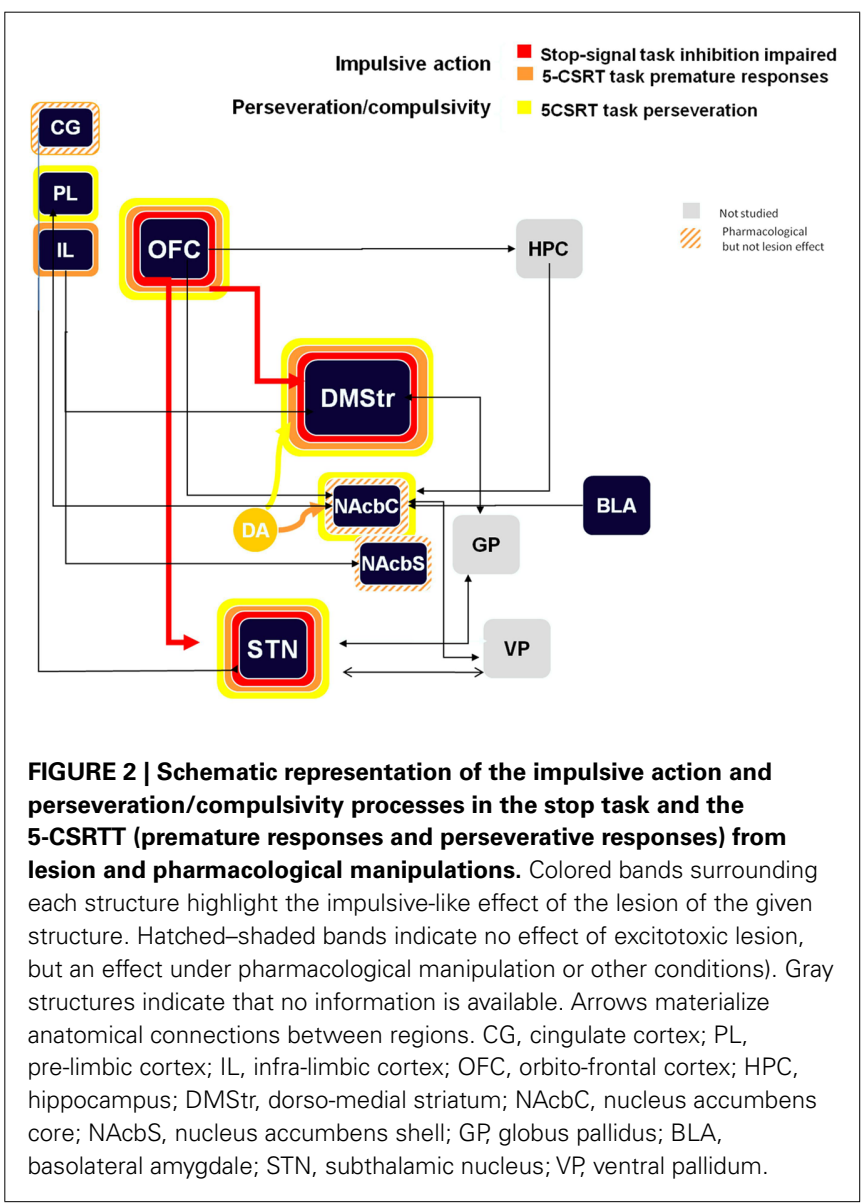




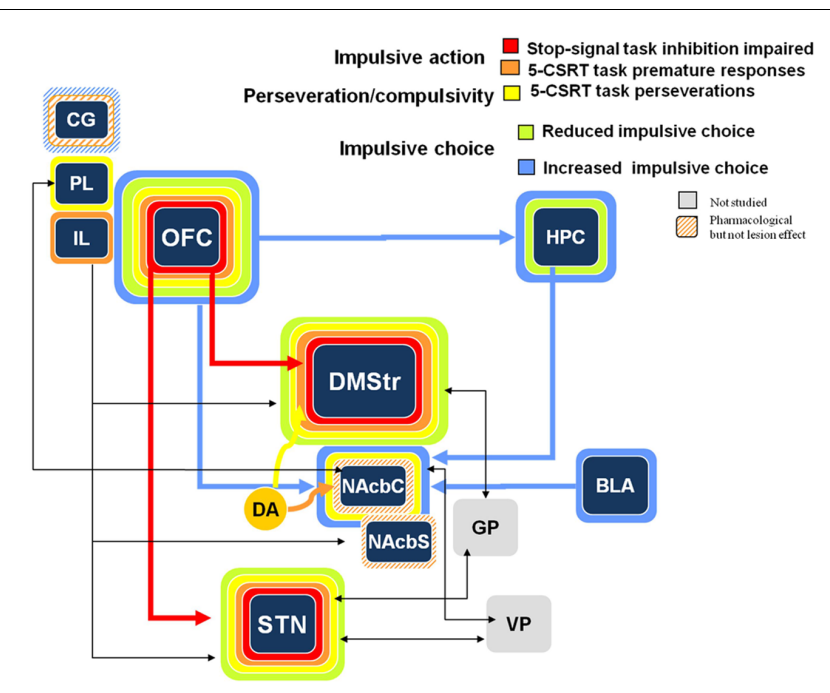

FIGURE 3 | Schematic representation of the impulsive action, perseveration/compulsivity, and impulsive choice processes in the stop task, the 5-CSRTT (premature responses and perseverative responses) and the delay-discounting task from lesion and pharmacological manipulations. Colored bands surrounding each structure highlight the impulsive-like effect of the lesion of the given structure. Hatched-shaded bands indicate no effect of excitotoxic lesion, but an effect under pharmacological manipulation or other conditions). Gray structures indicate that no information is available. Arrows materialize anatomical connections between regions. $\mathrm{CG}$, cingulate cortex; $\mathrm{PL}$, pre-limbic cortex; IL, infra-limbic cortex; OFC, orbito-frontal cortex; HPC, hippocampus; DMStr, dorso-medial striatum; NAcbC, nucleus accumbens core; NAcbS, nucleus accumbens shell; GP, globus pallidus; BLA, basolateral amygdale; STN, subthalamic nucleus; VP, ventral pallidum (from Eagle and Baunez, 2010)

rats. Indeed, in the 5-CSRTT, bilateral lesions of the STN dramatically increases the number of perseverative responses made at the food receptacle (Baunez and Robbins, 1997). This deficit may indicate an increase in motivation for the reward. In addition, clinical reports showed that an infarct or tumor in the STN could lead to pathology such as hyperphagia, hypersexuality, and mood exaltation (Trillet et al., 1995; Absher et al., 2000; Barutca et al., 2003). Taken together, these data suggest that the STN may be implicated in motivation.

We thus decided to assess directly the role of the STN in motivational processes by studying its involvement in motivation for food (Baunez et al., 2002). In this study, we first showed that bilateral lesions of the STN did not affect primary motivational processes as assessed by measures of food consumption during 2 or $24 \mathrm{~h}$. On the other hand, STN lesions increased motivation for stimuli associated with food as assessed in several behavioral tasks. The conditioned locomotor activity procedure allows measurement of locomotor activity when animals anticipate food delivery. After conditioning, rats with STN lesions had a higher locomotor activity during the waiting period that indicates a higher level of expectation for the food. Furthermore, in a conditioned reinforcement task, in which a lever is associated with a conditioned stimulus (CS, i.e., a light previously paired with food), when compared to control rats performance, STN lesions increased the number of responses on the lever associated with the CS. These results show that STN lesions do not affect the association between the CS and the unconditioned stimulus (US, i.e., the food), but that it increases the motivation for the reward. Further studies looking at the role of the STN in food motivation confirmed these results using other behavioral tasks. Indeed, in a conditioned place preference task, STN lesions increased the time spent in the compartment associated with food (Baunez et al., 2005). In this paradigm, animals learn to associate a particular environment with a particular reward, while another environment serves as control. After conditioning, in the absence of the reward, an increase in the time spent in the environment associated with the reward indicates an increase in the incentive motivation for it. In addition, in an autoshaping or a sign tracking task, in which a visual stimulus or a lever is presented as a CS when the reward is delivered, STN lesions increased the number of approaches to the stimulus or lever presses on the lever associated with reward delivery and not on the control lever (Winstanley et al., 2005; Uslaner et al., 2008), thus confirming the increased motivation for stimuli associated with food after STN lesion (Baunez et al., 2002). Finally, although the food consumption measured in a continuous schedule of reinforcement (fixed ratio 1, FR1), in which one lever press leads to the delivery of one sucrose pellet, is not affected by STN lesion (Baunez et al., 2002), the willingness to work for food was increased by STN lesions as shown in the progressive ratio task where the lesion increases the last ratio completed (Baunez et al., 2005). In this task, the number of lever presses required to receive the same reward increases within the session until the animal stops responding for the reward, thereby reaching its breaking point (or last ratio completed). This paradigm allows direct measurement of the motivation to exert effort to obtain a particular reward. It is possible that the STN lesion impaired the perception of the "cost of the reward" as observed after excitotoxic lesion of the nucleus accumbens (Bowman and Brown, 1998). However, this could hardly explain the results observed in the conditioned place preference since this task does not involve any effort or cost.

Taken together these results show that the STN is implicated in incentive motivation for food since lesions to this nucleus increase responses to stimuli associated with food. In contrast, the STN is not involved in food consumption, ruling out the possibility that STN lesions might simply increase hunger.

Interestingly, further studies have shown that STN lesions do not affect cocaine or alcohol consumption either, as measured in a FR1 task (Baunez et al., 2005; Lardeux and Baunez, 2008). The lesion does not change alcohol consumption in the home cage, whatever the alcohol concentration tested or the type of alcohol [ethanol or aniseed-flavored alcohol (i.e., pastis); Lardeux and Baunez, 2008]. These results show that whatever the reward tested, the lesion of the STN does not affect reward consumption, which rules out a possible accidental lesion of the lateral hypothalamus that is in close vicinity with the STN. The STN is thus not involved in primary reward processes.

Interestingly, as for food, STN lesions affect motivational processes for cocaine and alcohol, but in different ways. Indeed, a striking result shows that STN lesions decrease motivation for cocaine, having an opposite effect than for food (Baunez et al., 2005). In fact, STN lesions decrease the willingness to work for self-administered cocaine, as assessed in the progressive ratio task. 
It is to be noted that another study showed the opposite results (Uslaner et al., 2005), but number of reward the rats received per session is so low that one may wonder whether or not the animals had acquired the self-administration behavior. Furthermore, in the conditioned place preference task, lesions of the STN decreased the time spent in the compartment associated with cocaine. These unexpected results highlight a possible role for the STN in modulating the reactivity of the reward system with regard to the nature of the reward involved, and open a new and interesting research strategy for treatment of cocaine addiction. Indeed, the STN could be an interesting target where motivation for the drug could be decreased, while other forms of motivation would not be altered, and even a resumption of interest in some activities would occur. To explore this possibility further, we then tried to replicate these results using DBS of the STN instead of a lesion. Interestingly, as for the lesion and shown in Figure 4, STN HFS decreases the motivation for cocaine while increasing the motivation for food (Rouaud et al., 2010). These results, while obtained in intact rats, are in line with some clinical observations in PD patients, reporting craving for sweet food in some cases, or decreased addictive behavior toward their dopaminergic treatment after STN DBS (Witjas et al., 2005; Ardouin et al., 2006; Knobel et al., 2008; Lim et al., 2009). These results suggest that STN HFS could thus represent an interesting strategy for the treatment of cocaine addiction.

When testing the effects of bilateral STN lesions on motivation for alcohol, we have shown that it could also affect motivation in an opposite manner depending on the animals' initial preference for the reward (Lardeux and Baunez, 2008). In this study, rats were separated into two groups, High and Low Drinkers depending on their alcohol preference. After STN lesions, High Drinker rats showed an increase of their motivation for alcohol, while Low Drinker rats showed a decrease in their motivation, as assessed by both the progressive ratio task and the conditioned place preference task (Lardeux and Baunez, 2008).

Taken together, these behavioral results show that STN lesions affect incentive motivation depending on the nature of the reward, but also on the initial level of preference for the reward. This latter observation highlights how critical pre-surgery assessment of the patients can be, in order to prevent or better anticipate some of the possible side-effects of STN DBS in PD patients (for example, in a patient with history of alcoholism, STN DBS could lead to relapse).

The first motivational neuronal correlates in the STN have been observed in the monkey (Matsumura et al., 1992). They recorded STN neurons that were activated only when the target presentation was followed by reward delivery. A more recent monkey study showed that STN neurons respond during the reward anticipation phase or after the reward delivery (Darbaky et al., 2005). In the rat, neuronal responses to the stimulus predicting the reward and to the reward delivery have also been shown (Teagarden and Rebec, 2007). We have recently performed electrophysiological recording of STN neurons in rats revealing that they can encode the value of the reward (Lardeux et al., 2009). We designed a task where the rat had to press and hold a lever down for $1 \mathrm{~s}$. Halfway through the holding period, a stimulus predicting which one of the two possible rewards will

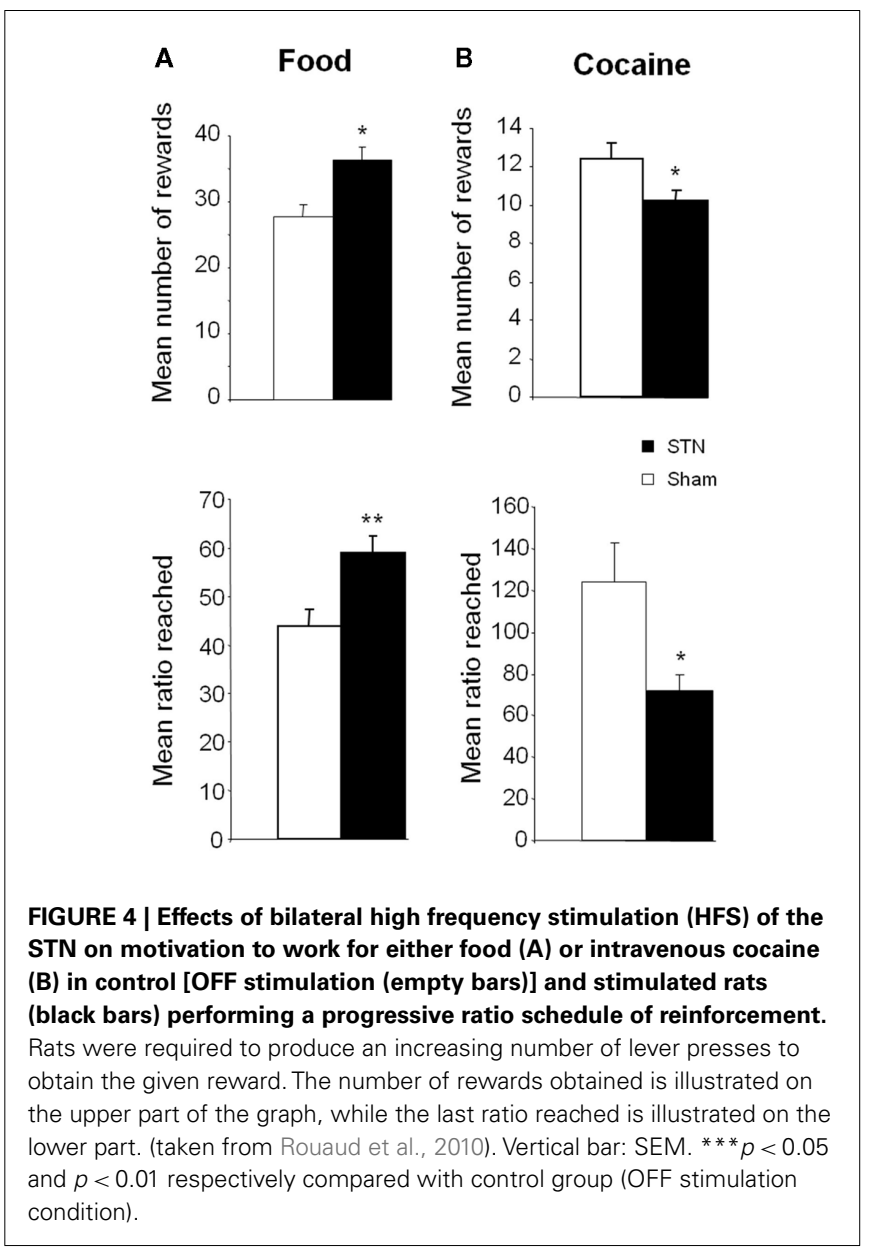

be delivered at the end of the trial was briefly presented. We have shown that STN neurons could be categorized into subpopulations responding differently to the various rewards. One sub-population responded exclusively to the cue predicting a $4 \%$ sucrose solution, but did not respond to the cue predicting the other reward (32\% sucrose solution). The other sub-population responded to the cue predicting $32 \%$ sucrose, but not to the cue predicting 4\% (Lardeux et al., 2009). In another study, we further showed that this dissociation is also observed when the two rewards are sucrose and cocaine (Lardeux et al., 2008). These studies showed that the STN encodes the reinforcing properties of a reward. We have also shown that some STN neurons, that we called "oops" neurons, encode behavioral error depending on the expected reward.

These electrophysiological results showing a differential encoding depending on the nature and on the preference for a reward are in line with the behavioral results suggesting a role of STN in motivation with regards to the nature of the reward, its valence and also to the preference for it. Since the encoding of the relative preference for a reward has been observed in the OFC of the monkey (Tremblay and Schultz, 1999), it might well be possible that this role of STN is under the control of the OFC via the so-called "hyperdirect pathway." The direct cortical influence on STN activity may also explain the existence of "oops neurons" that 
share properties with anterior cingulate neurons recorded in the monkey (Amiez et al., 2005).

\section{GENERAL CONCLUSION}

By reviewing the wide-range of data available regarding the involvement of the STN in frontal-like functions, it is now obvious that this small region of the brain plays a critical role in far more than motor functions. Not only can inactivation of the STN affect attentional processes, it can induce a loss of inhibitory control, in line with the proposition that an intact STN could help "holding your horses" (Frank et al., 2007) and affect motivational processes. These possible dysfunctions caused by STN inactivation could be important when considering that the current strategy for the treatment of parkinsonism is STN DBS. Whether STN DBS mimics strictly an inactivation is still under debate, but several lines of evidence indicate that it might not be truly the case (see for review Gubellini et al., 2009).

For many years the STN has been considered as a relay structure of the striatal information on the so-called "indirect pathway." The network we have highlighted involving the dorso-medial striatum supports this view. However, it also seems obvious from various points raised in this review that the hyperdirect pathway, especially that involving the OFC, contributes a lot to some of

\section{REFERENCES}

Absher, J. R., Vogt, B. A., Clark, D. G., Flowers, D. L., Gorman, D. G., Keyes, J. W., and Wood, F. B. (2000). Hypersexuality and hemiballism due to subthalamic infarction. Neuropsychiatry Neuropsychol. Behav. Neurol. $13,220-229$.

Alexander, G. E., DeLong, M. R., and Strick, P. L. (1986). Parallel organization of functionally segregated circuits linking basal ganglia and cortex. Annu. Rev. Neurosci. 9, 357-381.

Amiez, C., Joseph, J. P., and Procyk, E. (2005). Anterior cingulate errorrelated activity is modulated by predicted reward 2. Eur. J. Neurosci. 21, 3447-3452.

Ardouin, C., Pillon, B., Peiffer, E., Bejjani, P., Limousin, P., Damier, P., Arnulf, I., Benabid, A. L., Agid, Y., and Pollak, P. (1999). Bilateral subthalamic or pallidal stimulation for Parkinson's disease affects neither memory nor executive functions: a consecutive series of 62 patients. Ann. Neurol. 46, 217-223.

Ardouin, C., Voon, V., Worbe, Y., Abouazar, N., Czernecki, V., Hosseini, H., Pelissolo, A., Moro, E., Lhommee, E., Lang, A. E., Agid, Y., Benabid, A. L., Pollak, P., Mallet, L., and Krack, P. (2006). Pathological gambling in Parkinson's disease improves on chronic subthalamic nucleus stimulation. Mov. Disord. 21, 1941-1946.
Aron, A. R., and Poldrack, R. A. (2006). Cortical and subcortical contributions to stop signal response inhibition: role of the subthalamic nucleus. J. Neurosci. 26, 2424-2433.

Ballanger, B., van, E. T., Moro, E., Lozano, A. M., Hamani, C., Boulinguez, P., Pellecchia, G., Houle, S., Poon, Y. Y., Lang, A. E., and Strafella, A. P. (2009). Stimulation of the subthalamic nucleus and impulsivity: release your horses. Ann. Neurol. 66, 817-824.

Barutca, S., Turgut, M., Meydan, N., and Ozsunar, Y. (2003) Subthalamic nucleus tumor causing hyperphagia - case report. Neurol. Med. Chir. (Tokyo) 43, 457-460.

Baunez, C., Amalric, M., and Robbins, T. W. (2002). Enhanced food-related motivation after bilateral lesions of the subthalamic nucleus. J. Neurosci. $22,562-568$.

Baunez, C., Christakou, A., Chudasama, Y., Forni, C., and Robbins, T. W. (2007). Bilateral highfrequency stimulation of the subthalamic nucleus on attentional performance: transient deleterious effects and enhanced motivation in both intact and parkinsonian rats. Eur. J. Neurosci. 25, 1187-1194.

Baunez, C., Dias, C., Cador, M., and Amalric, M. (2005). The subthalamic nucleus exerts opposite control on cocaine and "natural" rewards. Nat. Neurosci. 8, 484-489.

the behavioral responses observed after STN manipulation. To what extent and how the STN integrates both direct cortical and indirect striatal informations remains to be elucidated, but the STN may be at a critical juncture to operate as a "gate-control" for action selection under the influence of the most valuable reward.

Despite its relatively small size, the STN appears to be not only important and valuable for the treatment of PD, but its involvement in frontal functions suggests that it could be an interesting structure to target for the treatment of psychiatric disorders such as OCD (Mallet et al., 2008) and possibly addiction (Krack et al., 2010; Rouaud et al., 2010).

\section{ACKNOWLEDGMENTS}

This work has been supported by grants from the "Centre National de la Recherche Scientifique" (CNRS), the "Université de Provence," the "Agence Nationale pour la Recherche" (ANR-05JC05_48262 and ANR-09-MNPS-028-01 to Christelle Baunez), the MILDT-InCa-INSERM grant to Christelle Baunez, the Fondation pour la Recherche sur le Cerveau to Christelle Baunez and the Ministry for Higher Education and Research to Sylvie Lardeux. The authors thank Dr. L. Mallet for helpful suggestions on the manuscript and Dr. C. Winstanley for improving the english.

Baunez, C., Nieoullon, A., and Amalric, M. (1995) In a rat model of parkinsonism, lesions of the subthalamic nucleus reverse increases of reaction time but induce a dramatic premature responding deficit. J. Neurosci. 15, 6531-6541.

Baunez, C., and Robbins, T. W. (1997). Bilateral lesions of the subthalamic nucleus induce multiple deficits in an attentional task in rats. Eur. J. Neurosci. 9, 2086-2099.

Baunez, C., and Robbins, T. W. (1999). Effects of transient inactivation of the subthalamic nucleus by local muscimol and APV infusions on performance on the five-choice serial reaction time task in rats. Psychopharmacology (Berl.) 141, 57-65.

Benazzouz, A., Gross, C., Feger, J. Boraud, T., and Bioulac, B. (1993). Reversal of rigidity and improvement in motor performance by subthalamic high-frequency stimulation in MPTP-treated monkeys. Eur. J. Neurosci. 5, 382-389.

Bergman, H., Wichmann, T., and DeLong, M. R. (1990). Reversal of experimental parkinsonism by lesions of the subthalamic nucleus. Science 249, 1436-1438.

Bowman, E. M., and Brown, V. J. (1998) Effects of excitotoxic lesions of the rat ventral striatum on the perception of reward cost 4. Exp. Brain Res. 123, 439-448.

Brusa, L., Pierantozzi, M., Peppe, A., Altibrandi, M. G., Giacomini,
P., Mazzone, P., and Stanzione, P. (2001). Deep brain stimulation (DBS) attentional effects parallel those of l-dopa treatment. J. Neural Transm. 108, 1021-1027.

Chudasama, Y., Baunez, C., and Robbins, T. W. (2003). Functional disconnection of the medial prefrontal cortex and subthalamic nucleus in attentional performance: evidence for corticosubthalamic interaction. J. Neurosci. 23, 5477-5485.

Darbaky, Y., Baunez, C., Arecchi, P., Legallet, E., and Apicella, P. (2005). Reward-related neuronal activity in the subthalamic nucleus of the monkey. Neuroreport 16, 1241-1244.

Desbonnet, L., Temel, Y., VisserVandewalle, V., Blokland, A. Hornikx, V., and Steinbusch, H. W. (2004). Premature responding following bilateral stimulation of the rat subthalamic nucleus is amplitude and frequency dependent. Brain Res. 1008, 198-204.

Eagle, D. M., Bari, A., and Robbins, T. W. (2008a). The neuropsychopharmacology of action inhibition: cross-species translation of the stop-signal and go/no-go tasks. Psychopharmacology (Berl.) 199, 439-456.

Eagle, D. M., Baunez, C., Hutcheson, D. M., Lehmann, O., Shah, A. P., and Robbins, T. W. (2008b). Stop-signal reaction-time task performance: role of prefrontal cortex and subthalamic nucleus. Cereb. Cortex 18, 178-188. 
Eagle, D. M., and Baunez, C. (2010). Is there an inhibitory-responsecontrol system in the rat? Evidence from anatomical and pharmacological studies of behavioral inhibition. Neurosci. Biobehav. Rev. 34, 50-72.

Fimm, B., Heber, I. A., Coenen, V. A., Fromm, C., Noth, J., and Kronenbuerger, M. (2009). Deep brain stimulation of the subthalamic nucleus improves intrinsic alertness in Parkinson's disease. Mov. Disord. 24, 1613-1620.

Frank, M. J., Samanta, J., Moustafa, A. A., and Sherman, S. J. (2007). Hold your horses: impulsivity, deep brain stimulation, and medication in parkinsonism. Science 318, 1309-1312.

Groenewegen, H. J., and Berendse, H. W. (1990). Connections of the subthalamic nucleus with ventral striatopallidal parts of the basal ganglia in the rat. J. Comp. Neurol. 294, 607-622.

Groenewegen, H. J., Berendse, H. W., and Haber, S. N. (1993). Organization of the output of the ventral striatopallidal system in the rat: ventral pallidal efferents. Neuroscience 57, 113-142.

Gubellini, P., Salin, P., Kerkerian-LeGoff, L., and Baunez, C. (2009). Deep brain stimulation in neurological diseases and experimental models: from molecule to complex behavior. Prog. Neurobiol. 89, 79-123.

Hershey, T., Campbell, M. C., Videen, T. O., Lugar, H. M., Weaver, P. M., Hartlein, J., Karimi, M., Tabbal, S. D., and Perlmutter, J. S. (2010). Mapping Go-No-Go performance within the subthalamic nucleus region. Brain 133, 3625-3634.

Hershey, T., Revilla, F. J., Wernle, A. R., Gee-Minnich, L., Antenor, J. V., Videen, T. O., Dowling, J. L., Mink, J. W., and Perlmutter, J. S. (2003). Cortical and subcortical blood flow effects of subthalamic nucleus stimulation in PD. Neurology 61, 816-821.

Knobel, D., Aybek, S., Pollo, C., Vingerhoets, F. J., and Berney, A. (2008). Rapid resolution of dopamine dysregulation syndrome (DDS) after subthalamic DBS for Parkinson disease (PD): a case report. Cogn. Behav. Neurol. 21, 187-189.

Krack, P., Hariz, M. I., Baunez, C., Guridi, J., and Obeso, J. A. (2010). Deep brain stimulation: from neurology to psychiatry? 1. Trends Neurosci. 33, 474-484.

Lardeux, S., and Baunez, C. (2008). Alcohol preference influences the subthalamic nucleus control on motivation for alcohol in rats. $\mathrm{Neu}$ ropsychopharmacology 33, 634-642.
Lardeux, S., Paleressompoulle, D., Pernaud, R., Cador, M., and Baunez, C. (2008). Selective encoding of natural reward versus cocaine by subthalamic nucleus neurons. Abstr. Soc. Neurosci. 34, 88.4.

Lardeux, S., Pernaud, R., Paleressompoulle, D., and Baunez, C. (2009). Beyond the reward pathway: coding reward magnitude and error in the rat subthalamic nucleus. J. Neurophysiol. 102, 2526-2537.

Le Jeune, F., Verin, M., N'Diaye, K., Drapier, D., Leray, E., du Montcel, S. T., Baup, N., Pelissolo, A., Polosan, M., Mallet, L., Yelnik, J., Devaux, B., Fontaine, D., Chereau, I. Bourguignon, A., Peron, J., Sauleau, P., Raoul, S., Garin, E., Krebs, M. O., Jaafari, N., and Millet, B. (2010). Decrease of prefrontal metabolism after subthalamic stimulation in obsessive-compulsive disorder: a positron emission tomography study. Biol. Psychiatry 68, 1016-1022.

Lim, S. Y., O’Sullivan, S. S., Kotschet, K., Gallagher, D. A., Lacey, C., Lawrence, A. D., Lees, A. J., O'Sullivan, D. J., Peppard, R. F., Rodrigues, J. P., Schrag, A., Silberstein, P., Tisch, S., and Evans, A. H. (2009). Dopamine dysregulation syndrome, impulse control disorders and punding after deep brain stimulation surgery for Parkinson's disease. J. Clin. Neurosci. 16, 1148-1152.

Limousin, P., Pollak, P., Benazzouz, A., Hoffmann, D., Le Bas, J. F., Broussolle, E., Perret, J. E., and Benabid, A. L. (1995). Effect of parkinsonian signs and symptoms of bilateral subthalamic nucleus stimulation. Lancet 345, 91-95.

Lindsley, D. F., Barton, R. J., and Atkins, R. J. (1970). Effects of subthalamic lesions on peripheral and central arousal thresholds in cats. Exp. Neurol. 26, 109-119.

Mallet, L., Polosan, M., Jaafari, N., Baup, N., Welter, M. L., Fontaine, D., du Montcel, S. T., Yelnik, J., Chereau, I., Arbus, C., Raoul, S., Aouizerate, B., Damier, P., Chabardes, S., Czernecki, V., Ardouin, C., Krebs, M. O., Bardinet, E., Chaynes, P., Burbaud, P., Cornu, P., Derost, P., Bougerol, T., Bataille, B., Mattei, V., Dormont, D., Devaux, B., Verin, M., Houeto, J. L., Pollak, P., Benabid, A. L., Agid, Y., Krack, P., Millet, B., and Pelissolo, A. (2008) Subthalamic nucleus stimulation in severe obsessive-compulsive disorder. $\mathrm{N}$. Engl. J. Med. 359, 2121-2134.

Mallet, L., Schupbach, M., N'Diaye, K., Remy, P., Bardinet, E., Czernecki, V., Welter, M. L., Pelissolo, A.,
Ruberg, M., Agid, Y., and Yelnik, J. (2007). Stimulation of subterritories of the subthalamic nucleus reveals its role in the integration of the emotional and motor aspects of behavior. Proc. Natl. Acad. Sci. U.S.A. 104, 10661-10666.

Matsumura, M., Kojima, J., Gardiner, T W., and Hikosaka, O. (1992). Visual and oculomotor functions of monkey subthalamic nucleus. J. Neurophysiol. 67, 1615-1632.

Maurice, N., Deniau, J. M., Glowinski, J., and Thierry, A. M. (1998a) Relationships between the prefrontal cortex and the basal ganglia in the rat: physiology of the corticosubthalamic circuits. J. Neurosci. 18, 9539-9546.

Maurice, N., Deniau, J. M., Menetrey, A., Glowinski, J., and Thierry, A. M. (1998b). Prefrontal cortexbasal ganglia circuits in the rat: involvement of ventral pallidum and subthalamic nucleus. Synapse 29 , 363-370.

Maurice, N., Deniau, J. M., Glowinski, J., and Thierry, A. M. (1999). Relationships between the prefrontal cortex and the basal ganglia in the rat: physiology of the corticonigral circuits. J. Neurosci. 19 4674-4681.

Nambu, A., Tokuno, H., and Takada, M. (2002). Functional significance of the cortico-subthalamo-pallidal "hyperdirect" pathway. Neurosci. Res. 43, 111-117.

Naquet, R., Denavit, M., and be-Fessard D. (1966). Comparison between the role of the subthalamus and that of the different bulbomesencephalic structures in the maintenance of wakefulness. Electroencephalogr. Clin. Neurophysiol. 20, 149-164.

Phillips, J. M., and Brown, V. J. (1999). Reaction time performance following unilateral striatal dopamine depletion and lesions of the subthalamic nucleus in the rat. Eur. J. Neurosci. 11, 1003-1010.

Robbins, T. W. (2002). The 5choice serial reaction time task: behavioural pharmacology and functional neurochemistry. Psychopharmacology (Berl.) 163, 362-380.

Rouaud, T., Lardeux, S., Panayotis, N., Paleressompoulle, D., Cador, M., and Baunez, C. (2010). Reducing the desire for cocaine with subthalamic nucleus deep brain stimulation. Proc. Natl. Acad. Sci. U.S.A. 107, 1196-1200.

Rubia, K., Russell, T., Overmeyer, S., Brammer, M. J., Bullmore, E. T. Sharma, T., Simmons, A., Williams,
S. C., Giampietro, V., Andrew, C. M., and Taylor, E. (2001). Mapping motor inhibition: conjunctive brain activations across different versions of go/no-go and stop tasks. Neuroimage 13, 250-261.

Saint-Cyr, J. A., Trepanier, L. L., Kumar, R., Lozano, A. M., and Lang, A. E. (2000). Neuropsychological consequences of chronic bilateral stimulation of the subthalamic nucleus in Parkinson's disease. Brain 123(Pt 10), 2091-2108.

Teagarden, M. A., and Rebec, G. V. (2007). Subthalamic and striatal neurons concurrently process motor, limbic, and associative information in rats performing an operant task. J. Neurophysiol. 97, 2042-2058.

Temel, Y., Blokland, A., Steinbusch, H. W., and Visser-Vandewalle, V. (2005). The functional role of the subthalamic nucleus in cognitive and limbic circuits 2. Prog. Neurobiol. 76, 393-413.

Tremblay, L., and Schultz, W. (1999). Relative reward preference in primate orbitofrontal cortex. Nature 398, 704-708.

Trillet, M., Vighetto, A., Croisile, B., Charles, N., and Aimard G. (1995). Hemiballismus with logorrhea and thymoaffective disinhibition caused by hematoma of the left subthalamic nucleus. Rev. Neurol. (Paris) 151, 416-419.

Uslaner, J. M., Dell'Orco, J. M., Pevzner, A., and Robinson, T. E. (2008). The influence of subthalamic nucleus lesions on sign-tracking to stimuli paired with food and drug rewards: facilitation of incentive salience attribution? Neuropsychopharmacology 33, 2352-2361.

Uslaner, J. M., and Robinson, T. E. (2006). Subthalamic nucleus lesions increase impulsive action and decrease impulsive choice - mediation by enhanced incentive motivation? Eur. J. Neurosci. 24, 2345-2354.

Uslaner, J. M., Yang, P., and Robinson, T. E. (2005). Subthalamic nucleus lesions enhance the psychomotor-activating, incentive motivational, and neurobiological effects of cocaine 1. J. Neurosci. 25, 8407-8415.

Winstanley, C. A., Baunez, C., Theobald, D. E., and Robbins, T. W. (2005). Lesions to the subthalamic nucleus decrease impulsive choice but impair autoshaping in rats: the importance of the basal ganglia in Pavlovian conditioning and impulse control. Eur. J. Neurosci. 21, 3107-3116. 
Witjas, T., Baunez, C., Henry, J. M., Delfini, M., Regis, J., Cherif, A. A., Peragut, J. C., and Azulay, J. P. (2005). Addiction in Parkinson's disease: impact of subthalamic nucleus deep brain stimulation. Mov. Disord. 20, 1052-1055.

York, M. K., Dulay, M., Macias, A., Levin, H. S., Grossman, R., Simpson, R., and Jankovic, J. (2008). Cognitive declines following bilateral subthalamic nucleus deep brain stimulation for the treatment of Parkinson's disease. J. Neurol. Neurosurg. Psychiatr. 79, 789-795.

Conflict of Interest Statement: The authors declare that the research was conducted in the absence of any commercial or financial relationships that could be construed as a potential conflict of interest.

Received: 22 April 2011; paper pending published: 25 July 2011; accepted: 21 September 2011; published online: 11 October 2011.

Citation: Baunez $C$ and Lardeux $S$ (2011) Frontal cortex-like functions of the subthalamic nucleus.
Front. Syst. Neurosci. 5:83. doi: 10.3389/fnsys.2011.00083

Copyright (C) 2011 Baunez and Lardeux. This is an open-access article subject to a non-exclusive license between the authors and Frontiers Media SA, which permits use, distribution and reproduction in other forums, provided the original authors and source are credited and other Frontiers conditions are complied with. 